\title{
Linear sloshing frequencies in the annular region of a circular cylindrical container in the presence of a rigid baffle
}

\author{
N CHOUDHARY and S N BORA* \\ Department of Mathematics, Indian Institute of Technology Guwahati, Guwahati 781039, India \\ e-mail: neelam@iitg.ernet.in; swaroop@iitg.ernet.in
}

MS received 21 September 2015; revised 10 September 2016; accepted 30 November 2016

\begin{abstract}
Sloshing in any type of container may invite instability to it. If some part of the free liquid surface in the annular region of a specially designed circular cylindrical container is covered with an annular baffle, the natural frequencies and the response of the liquid in the container undergo a drastic change. A partly covered free surface shifts the natural frequency above and away from the control frequency of the vehicle, in which the liquid-filled container is placed, which results in the reduction of sloshing mass participating in the dynamic motion of the system. The fundamental natural frequency of an inviscid and incompressible liquid is determined for increasing width of the baffle that is attached to the outer tank wall on the free surface. It is observed that by increasing the width of the baffle, natural frequencies can be significantly increased. Investigations are also carried out for different values of Bond number, which depicts different states of surface tension, and for varying values of the part of the radius in the fluid region. It is also observed that by increasing the fluid height inside the container, the natural frequencies can be increased, which results in reduction of sloshing.
\end{abstract}

Keywords. Sloshing; cylindrical container; Bond number; surface tension; baffle.

\section{Introduction}

The violent behaviour of fluid inside a container/tank with free surface is known as sloshing. The presence of a free surface in partially filled liquid containers allows for a fluid motion relative to the containers. This phenomenon is referred to as 'liquid sloshing', which is generally caused by external tank excitation and may have a significant influence on the response of the container. Liquid sloshing is a key factor in vehicle movement problems. It is necessary to perform the movement in such a way that the liquid does not splash out of the container. Sloshing was first encountered in the control of guided missiles in the aerospace industry. In this application, it was found that sloshing in the fuel tanks could represent instabilities. Similar problems have been encountered in the control of aeroplanes. When the motion of any vehicle or structure containing liquid free surface is considered, then sloshing is the result of the resonant excitation of the tank liquid. Excitation with frequencies in the vicinity of the lowest natural frequency is of primary practical interest. Resonant free surface flows in tanks in aircraft, missiles and rockets have been the focus of extensive research. Liquid sloshing is also a severe problem when transporting liquid in containers both on sea and on land. Sloshing generates severe hydrodynamic loads that can be dangerous for structural

*For correspondence integrity and stability of rockets, satellites, ships, trucks and even stationary petroleum containers. The problem of sloshing remains as a great concern to aerospace, civil, mechanical and nuclear engineers, physicists, designers of road tankers and ship tankers and mathematicians. The movement of fluid in containers of different shapes and sizes has been investigated by a large number of researchers. A variety of container shapes exist such as rectangular, prismatic, tapered, spherical, horizontal/vertical cylindrical, etc. The liquid inside the container may be oil, liquefied gas or water. Analytical solutions are not possible for all types of geometries. Explicit solutions are possible only for a few special classes such as rectangular and cylindrical containers, which are usually used as containers/reservoirs in practical applications.

Resonant fluid sloshing in a rectangular tank has been studied theoretically, experimentally and numerically. A proper study of sloshing is required to estimate its effect in ship tanks in order to take into account this effect for transport of liquid. Ockendon et al [1] described an asymptotic approach to obtain multiple periodic solutions for resonant sloshing in shallow water. They assumed that the wave number appearing in dispersion relation was small and discussed dissipative effect. Evans [2] used a widespacing approximation to consider the scattering of waves from an arbitrary number of identical bodies and determined the resonant frequencies. He determined the resonant frequencies just for a single obstacle and then generalized it 
to $n$ symmetric obstacles by a compact expression for the $n$th power of a $2 \times 2$ matrix. Rocca et al [3] derived a fully nonlinear mathematical model by applying a variational method for sloshing in a rotating container. Faltinsen et al [4] carried out an investigation in which they studied the nonlinear sloshing in a partially filled rectangular tank. They used the Bateman-Luke variational principle and derived infinite-dimensional modal system of ordinary differential equations in terms of generalized time-dependent coordinates of free surface elevation and velocity potential. Faltinsen and Timokha [5] extended the work done in [4] and described surge and pitch excitations also. When $h / l>0.24$ (where $l$ is the tank breadth and $h$ is mean fluid depth) and depth is shallow, their theory does not match with the experimental results. Faltinsen and Timokha [6] repeated the work carried out in [5] with a revised modal system for a fluid in a rectangular tank with intermediate depth $(0.1 \leq h / l \leq 0.24)$. Faltinsen et al [7] modified their multimodal approach to handle threedimensional sloshing in a rectangular-base basin. Faltinsen et al [8] continued the work done in [7] and showed the validity of their results with experimental data. Thiagarajan et al [9] studied the sloshing in a rectangular tank and discussed the wave motion and pressure at various filled levels of tanks. Considering a two-layer fluid in a rectangular tank, Mackey and Cox [10] discussed the fluid oscillation in a horizontal direction. They used an asymptotic expansion method and derived a second-order ordinary differential equation, which was a reduced form of the extended forced Korteweg de Vries (efKdV) equation. Using the Melnikov theory they showed the existence of a class of periodic solutions.

Cylindrical containers are usually preferred as containers by fuel tank and oil tank designers. A reasonable number of researchers have been investigating sloshing in cylindrical containers. McIver and McIver [11] investigated the behaviour at low and high frequencies of a horizontal cylinder with side walls almost vertical. They found that the high-frequency limit of the modes depended on the angle of intersection of the container with the free surface. Bauer and Eidel [12] found that insertion of a baffle at free surface increases the frequencies. They numerically evaluated the response to the translational excitation, which showed the shifting of the resonance to higher values. Papaspyrou et al [13] investigated sloshing in a horizontal cylinder and developed a mathematical model in which the general solution of sloshing potential was written as a series expansion of the time function, and they obtained a semi-analytical solution, resulting in a system of linear ordinary differential equations. Gavrilyuk et al [14] examined sloshing in a vertical circular cylinder with a rigid baffle and found that sloshing is affected by the size and location of the baffle. Yuanjun et al [15] discussed nonlinear sloshing in a circular cylindrical tank under pitching excitation by using variational principle. The nonlinear equations with kinematics, dynamics and free surface boundary conditions through variation were derived and solved by a multiple-scale method.
Sinai [16] considered a two-layer fluid problem in a vertical cylinder and obtained a semi-analytical solution for the sloshing frequencies. Sciortino et al [17] described sloshing of a layered fluid system in a cylindrical tank with an arbitrary shape by introducing a Hamiltonian mathematical model and showed its validity by laboratory experiments.

There are some works carried out for sloshing related to structures other than rectangular and cylindrical tanks. Albright and Concus [18] calculated the small-amplitude periodic sloshing modes of a liquid in a vertical right circular cylinder with a concave spheroidal bottom where the whole free surface was not above the bottom. Bian et al [19] determined the natural frequencies of a liquid slug in a circular tube. Gavrilyuk et al [20] considered the sloshing problem in a circular conical tank and derived a nonlinear finite-dimensional asymptotic modal system.

The natural frequencies of a frictionless and incompressible liquid in an upright circular cylindrical container with a free liquid surface are well known. With the inclusion of liquid surface tension, they can be derived from the appropriate dispersion relation.

The effect of liquid sloshing on the dynamics and control of liquid transport, e.g., the terrestrial transport of liquid, oil and liquid natural gas in ships and fuel in aviation and astrodynamics, has motivated researchers, resulting in a wide range of research. The benefit of a partly covered free surface is that it increases the natural frequencies and decreases the sloshing mass participating in the dynamic motion of the system. We wish to determine the sloshing modes and sloshing frequencies in the annular region of a cylindrical container in which a rigid annular baffle is attached to the outer wall. We investigate how sloshing depends on the geometry and size of the container and also on that of the cover. We examine the effect of baffle width, height and surface tension on sloshing frequencies and the consequences.

Frequency in the lowest mode is the lowest and in all other higher modes it has significantly higher values, which do not have major effects on the stability of the system. Hence it is necessary to take care of frequency in the first mode, which we have investigated here. More details on significance of the lowest mode can be found in the book by Ibrahim [21].

\section{Statement and formulation of the problem}

An incompressible and inviscid fluid is assumed to perform irrotational motion in the annular region between two coaxial vertical circular cylinders with a common base. The radius of the inner cylinder is taken as $R_{1}$ and that of the outer cylinder as $R_{2}$. The annular region of the cylinder is filled with a liquid up to a constant height $h$. We introduce a rigid annular baffle at the free surface mounted on the cylindrical wall $r=R_{2}$ such that the width of the free surface becomes $\alpha R_{2}, 0 \leq \alpha \leq 1-\left(R_{1} / R_{2}\right)$. A cylindrical coordinate system $(r, \theta, z)$ is considered, in which $z$ is 
measured vertically upwards from the undisturbed water level $z=0$ with the origin on it (figure 1). It is to be noted that any such arrangement of partial covering of the free surface exhibits an increase of the natural frequencies as well as a reduction of the participating sloshing mass. The geometry considered in the problem is shown in figure 1 .

The boundary value problem (BVP) for the sloshing problem in the cylindrical container is given by Laplace's equation

$$
\nabla^{2} \Phi(r, \theta, z, t)=0, \quad R_{1}<r<R_{2}, \quad 0 \leq \theta \leq 2 \pi, \quad-h<z<0,
$$

i.e.,

$$
\begin{aligned}
& \frac{\partial^{2} \Phi}{\partial r^{2}}+\frac{1}{r} \frac{\partial \Phi}{\partial r}+\frac{1}{r^{2}} \frac{\partial^{2} \Phi}{\partial \theta^{2}}+\frac{\partial^{2} \Phi}{\partial z^{2}}=0, \quad R_{1}<r<R_{2}, \\
& 0 \leq \theta \leq 2 \pi, \quad-h<z<0,
\end{aligned}
$$

with the boundary wall conditions

$$
\frac{\partial \Phi}{\partial r}=0, \quad r=R_{1} \quad \text { and } \quad r=R_{2},
$$

and bottom boundary condition

$$
\frac{\partial \Phi}{\partial z}=0, \quad z=-h .
$$

In addition, there are impermeability conditions to be satisfied due to the presence of the baffle at the free surface:

$$
\frac{\partial \Phi}{\partial z}=0, \quad z=0, \quad R_{1}+R_{2} \alpha \leq r \leq R_{2} .
$$

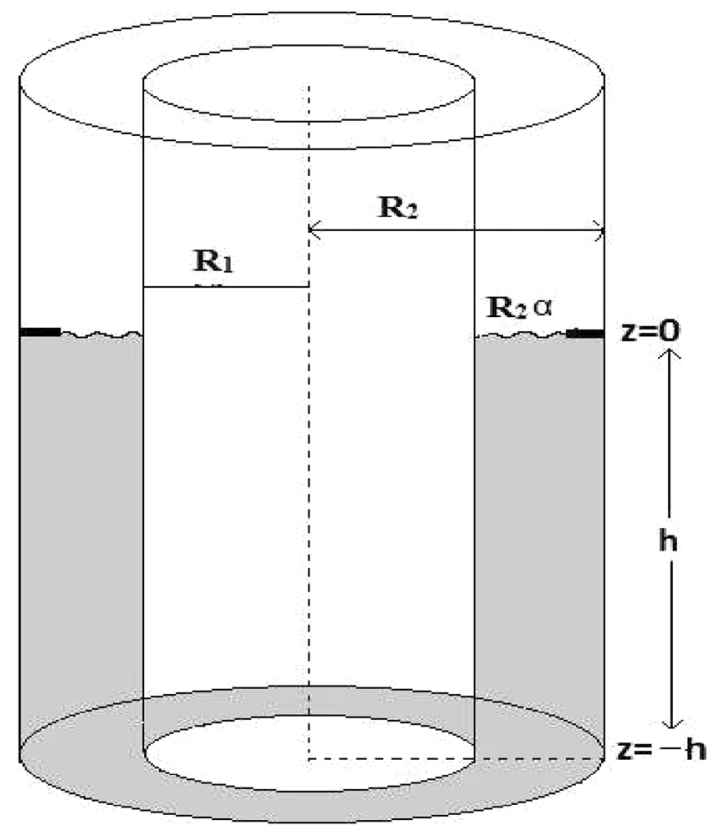

Figure 1. Schematic diagram for the problem.
The condition at $z=0$ in the range $R_{1}<r<R_{1}+R_{2} \alpha$ is obtained from the kinematic condition

$$
\frac{\partial \Phi}{\partial z}=\frac{\partial \eta}{\partial t},
$$

and the dynamic boundary condition with surface tension:

$$
\frac{\partial \Phi}{\partial t}+g \eta-\frac{\sigma}{\rho}\left[\frac{\partial^{2} \eta}{\partial r^{2}}+\frac{1}{r} \frac{\partial \eta}{\partial r}+\frac{1}{r^{2}} \frac{\partial^{2} \eta}{\partial \theta^{2}}\right]=\text { constant },
$$

where $\eta(r, \theta, z, t)$ is the free surface elevation, $\sigma$ the surface tension, $\rho$ the constant mass density and $g$ the gravitational constant. Equations (6) and (7) can be combined to get the free surface condition as

(ii) $\frac{\partial^{2} \Phi}{\partial t^{2}}+g \frac{\partial \Phi}{\partial z}+\frac{\sigma}{\rho} \frac{\partial^{3} \Phi}{\partial z^{3}}=0 \quad$ at $\quad z=0, \quad R_{1}<r<R_{1}+R_{2} \alpha$.

Due to the presence of the annular baffle at the wall, the upper surface condition is split as Eqs. (5) and (8), respectively, at the baffle and at the reduced free surface.

\section{Method of solution}

In order to solve the BVP, we apply the separation of variables method to governing equation (1) by assuming a solution of the form

$$
\Phi(r, \theta, z, t)=R(r) Z(z) \exp \{l(m \theta+\omega t)\} .
$$

We know that the governing equation gets converted to two ordinary differential equations-one each in $r$ and $z$, the solutions of which can be found as

$$
R(r)=A J_{m}(k r)+B Y_{m}(k r),
$$

and

$$
Z(z)=C \cosh k z+D \sinh k z,
$$

where $J_{m}(k r)$ and $Y_{m}(k r)$ are Bessel functions of the first and second kind, respectively, of order $m$.

Using boundary conditions (3) and (4), the velocity potential is obtained as

$$
\begin{aligned}
\Phi(r, \theta, z, t)= & \sum_{m=0}^{\infty} \sum_{n=1}^{\infty} A_{m n} \frac{\cosh \left\{\frac{k_{m n}}{R_{2}}(z+h)\right\}}{\cosh \left(\frac{k_{m n}}{R_{2}} h\right)} \\
& \left\{\frac{J_{m}\left(\frac{k_{m n}}{R_{2}} r\right) Y_{m}^{\prime}\left(k_{m n}\right)-J_{m}^{\prime}\left(k_{m n}\right) Y_{m}\left(\frac{k_{m n}}{R_{2}} r\right)}{Y_{m}^{\prime}\left(k_{m n}\right)}\right\} \\
& \times \exp \{l(m \theta+\omega t)\},
\end{aligned}
$$

where $k_{m n}$ are the zeros of 


$$
J_{m}^{\prime}\left(k_{m n} \frac{R_{1}}{R_{2}}\right) Y_{m}^{\prime}\left(k_{m n}\right)-J_{m}^{\prime}\left(k_{m n}\right) Y_{m}^{\prime}\left(k_{m n} \frac{R_{1}}{R_{2}}\right)=0,
$$

with ' denoting differentiation with respect to $r$. These zeros are evaluated based on a method devised by Sorolla et al [22]. The coefficients $A_{m n}$ and the angular frequency $\omega$ appearing in Eq. (10) are still not known. After introducing an annular baffle at the free surface mounted on the wall of the outer cylinder, for a fixed angular mode $m$, we choose $N_{1}+1$ points including the end points in $R_{1}+R_{2} \alpha \leq r \leq R_{2}$ at the free surface so as to get the impermeability condition split from the free surface condition as

$$
\begin{aligned}
& \sum_{n=1}^{N_{1}+N_{2}} \frac{A_{m n}}{Y_{m}\left(k_{m n}\right)} \frac{k_{m n}}{R_{2}} \tanh \left(k_{m n} \frac{h}{R_{2}}\right) \\
& \quad \times\left[J_{m}\left\{k_{m n}\left(\alpha+\frac{R_{1}}{R_{2}}+\frac{\left(1-\alpha-\frac{R_{1}}{R_{2}}\right) n_{1}}{N_{1}}\right)\right\}\right. \\
& \left.Y_{m}^{\prime}\left(k_{m n}\right)-Y_{m}\left\{k_{m n}\left(\alpha+\frac{R_{1}}{R_{2}}+\frac{\left(1-\alpha-\frac{R_{1}}{R_{2}}\right) n_{1}}{N_{1}}\right)\right\} J_{m}^{\prime}\left(k_{m n}\right)\right]=0
\end{aligned}
$$

for $n_{1}=0,1,2, \ldots . N_{1}$,

and choose $N_{2}-1$ points satisfying the reduced free surface condition

$$
\begin{aligned}
& \sum_{n=1}^{N_{1}+N_{2}} \frac{A_{m n}}{Y_{m}^{\prime}\left(k_{m n}\right)}\left[J_{m}\left\{k_{m n}\left(\frac{R_{1}}{R_{2}}+\frac{\alpha n_{2}}{N_{2}}\right)\right\} Y_{m}^{\prime}\left(k_{m n}\right)\right. \\
& \left.-Y_{m}\left\{k_{m n}\left(\frac{R_{1}}{R_{2}}+\frac{\alpha n_{2}}{N_{2}}\right)\right\} \times J_{m}^{\prime}\left(k_{m n}\right)\right] \\
& \times\left[\omega^{2}-\left\{g \frac{k_{m n}}{R_{2}}+\frac{\sigma}{\rho}\left(\frac{k_{m n}}{R_{2}}\right)^{3}\right\} \tanh \left(k_{m n} \frac{h}{R_{2}}\right)\right]=0
\end{aligned}
$$

for $n_{2}=1,2, \ldots . N_{2}-1$.

Equations (12) and (13) represent $N_{1}+N_{2}$ homogenous algebraic equations in the coefficients $A_{m 1}, A_{m 2}, \ldots$, $A_{m N_{1}+N_{2}}$. The condition of nonzero solution for a homogeneous system, due to truncation up to a finite number of points, gives rise to the frequency equation for the determination of sloshing frequencies of the liquid in the container. Equations (12) and (13) are obtained with the help of point allocation method. The Method of point allocation is a simple and widely used approach. In order to use baffle condition and free surface condition, $N_{1}+N_{2}$ equidistant points are chosen where first $N_{1}+1$ points satisfy baffle condition and the rest $N_{2}-1$ satisfy free surface condition. In this way, we have a homogenous linear system of $N_{1}+$ $N_{2}$ equations in $N_{1}+N_{2}$ unknowns involving the frequency parameter, which is to be determined. The condition of nontrivial solution for a homogeneous system leads to the requirement of zero determinant of the system, which gives us the value of required frequency. Basically, we calculate those values of frequency for which this system has a zero determinant. All numerical calculations are done in MATLAB.

\section{Numerical results and discussion}

Our main aim is to find the relevant frequency equation for different values of $R_{1}$ and $R_{2}$. After obtaining the semianalytical solution, we proceed to evaluate numerically the fundamental sloshing frequencies. For an annular baffle of width $w=R_{2}(1-\alpha)-R_{1}, 0<\alpha \leq 1-\left(R_{1} / R_{2}\right)$, covering the free surface, we consider different cases and determine the sloshing frequencies for the lowest mode $m$. We examine the effect of baffle width and liquid height as well as surface tension on sloshing frequencies. For $\alpha=1-\left(R_{1} / R_{2}\right)$, there is no baffle inserted at the free surface. We evaluate the fundamental frequency $\omega_{m n}^{*}$ by non-dimensionalizing the frequency equation as

$$
\omega_{m n}^{* 2}=\frac{\omega_{m n}^{2}}{g / R_{2}}=k_{m n}\left(1+\frac{k_{m n}^{2}}{B o}\right) \tanh \left(k_{m n} \frac{h}{R_{2}}\right),
$$

where the Bond number $B o=\left(\rho g R_{2}^{2}\right) / \sigma$ is taken as 100 for various $\alpha$ values. We evaluate frequencies for the angular mode $m=1$ and $n=1$, i.e., for $\omega_{11}^{*}$ which is determined from the values $k_{11}$ from Eq. (11). The lower modes are the modes that exhibit the larger sloshing mass participating in the slosh phenomenon.

\subsection{Case I: $R_{2} / R_{1}=2$, relevant upper surface conditions and numerical results}

Here we consider the radius of the inner cylinder as half of that of the outer cylinder, i.e., in this case $\alpha \in\left(0, \frac{1}{2}\right]$. Equations (12) and (13) are solved for $R_{2} / R_{1}=2$ and $\alpha \in\left(0, \frac{1}{2}\right]$.

Here we determine the dimensionless fundamental sloshing frequency as a function of liquid height ratio $h / R_{2}$ for a fixed Bond number and for various $\alpha$ values as shown in figure 2 . The curves shown in figure 2 are plotted for the lowest sloshing mode $\omega_{11}^{*}(m=1, n=1)$, which exhibits the largest sloshing mass participating in the motion. The curve for $w / R_{2}=0$ corresponds to the free surface in its entirety, i.e., there is no baffle on the free surface. Curves are drawn for various $\alpha$ values in increasing order of baffle width $w / R_{2}$.

We choose the intermediate value of Bond number $B o=\left(\rho g R_{2}^{2}\right) / \sigma=100$, which includes the effect of surface tension. A baffle with width of about $w=0.3 R_{2}$ doubles the fundamental natural frequency. For very small values of $h / R_{2}$, dimensionless frequency $\omega_{11}^{*}$ increases rapidly and then for higher values, it becomes stable. For the baffle width $w=0.4 R_{2}, \omega_{11}^{*}$ becomes twice as much as for the baffle width $w=0.3 R_{2}$. The value of $\omega_{11}^{*}$ for the baffle width $w=0.4 R_{2}$ is much higher as compared with the case where there is no baffle at the free surface. We observe that the natural frequency $\omega_{11}^{*}$ increases with increasing baffle width as well as increasing height ratio $h / R_{2}$.

Figure 3 shows the curves drawn for $\omega_{11}^{*}$ as a function of liquid height ratio $h / R_{2}$ for various $\alpha$ values in which surface tension $\sigma$ is assumed to be absent. It is observed that 


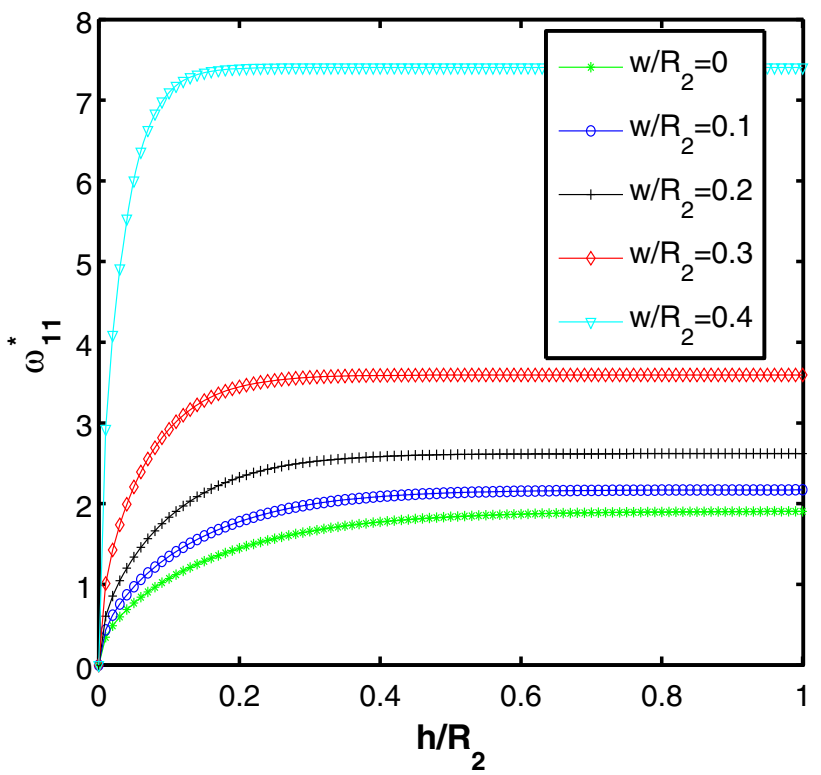

Figure 2. Sloshing frequency $\omega_{11}^{*}(m=1, n=1)$ for different annular baffles of width $\left(w=\left(\frac{1}{2}-\alpha\right) R_{2}\right)$ vs height ratio $h / R_{2}$ for $B o=100$

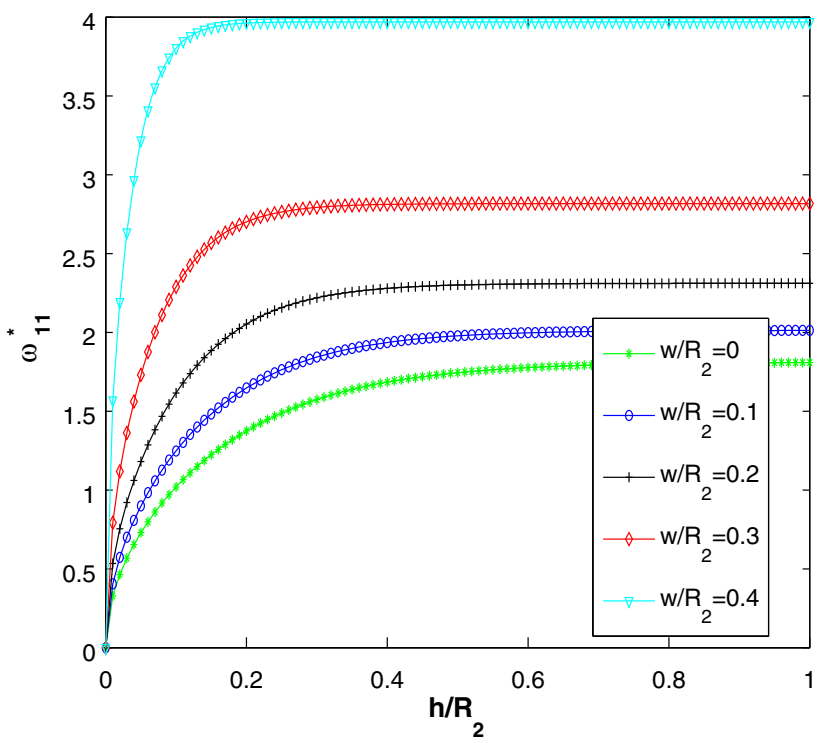

Figure 3. Sloshing frequency $\omega_{11}^{*}(m=1, n=1)$ for different annular baffles of width $\left(w=\left(\frac{1}{2}-\alpha\right) R_{2}\right)$ vs height ratio $h / R_{2}$ without surface tension $\sigma$.

for various $\alpha$ values, the effect of baffle width remains the same, resulting in reduced sloshing frequency. In the absence of surface tension, sloshing is observed to be more. For the annular baffle width $w=0.4 R_{2}, \omega_{11}^{*}$ reduces to nearly half as compared with $\omega_{11}^{*}$ with surface tension $\sigma$. It is observed that the natural frequency reduces in this case, i.e., without surface tension the natural frequency is less in comparison with what we get when Bond number $B o=$ 100 is considered.

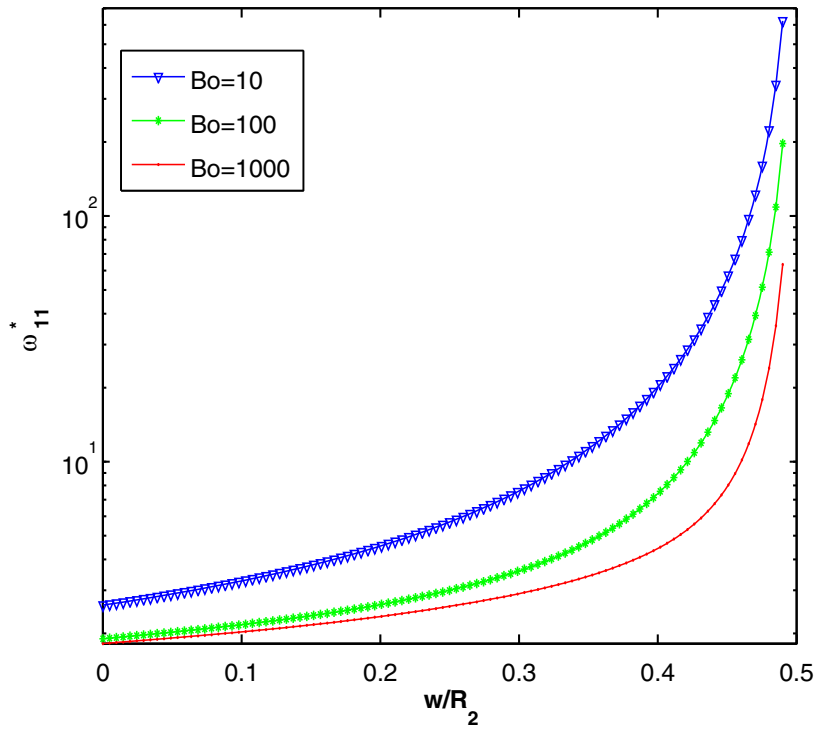

Figure 4. Effect of baffle width $w / R_{2}=\left(\frac{1}{2}-\alpha\right)$ on $\omega_{11}^{*}(m=$ $1, n=1)$ at height ratio $h / R_{2}=1$ for different Bond numbers.

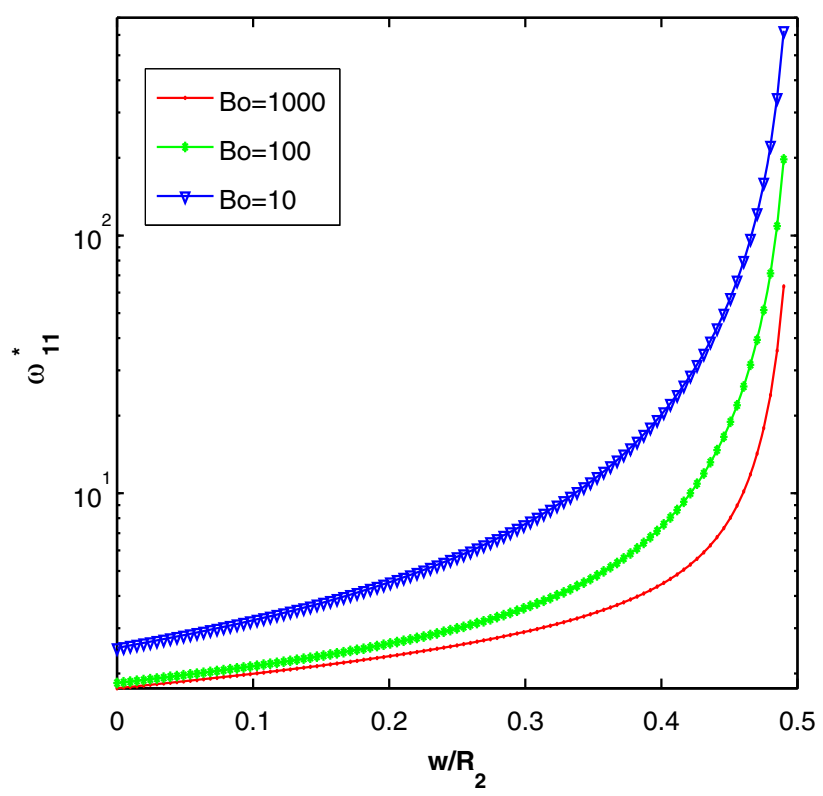

Figure 5. Effect of baffle width $w / R_{2}=\left(\frac{1}{2}-\alpha\right)$ on $\omega_{11}^{*}(m=$ $1, n=1)$ at height ratio $h / R_{2}=\frac{1}{2}$ for different Bond numbers.

In figures 4 and 5, we show the dimensionless fundamental sloshing frequency as a function of the magnitude of the annular baffle width $w / R_{2}=1 / 2-\alpha$ for fixed liquid height ratio $h / R_{2}=1,1 / 2$, respectively, for different Bond numbers $B o=10,100,1000$. Here it is observed that frequency increases when the value of Bond number decreases, i.e., when surface tension increases. Figures 2-5 establish that natural frequency increases with increasing baffle width, increasing height ratio and with decreasing Bond number. 


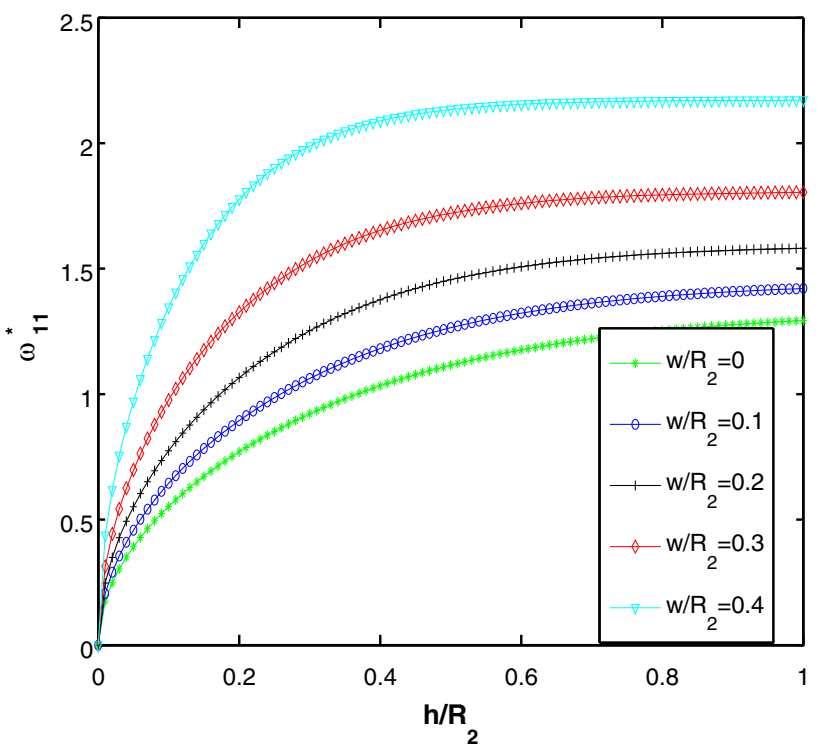

Figure 6. Sloshing frequency $\omega_{11}^{*}(m=1, n=1)$ for different annular baffles of width $\left(w=\left(\frac{2}{3}-\alpha\right) R_{2}\right)$ vs height ratio $h / R_{2}$ for $B o=100$

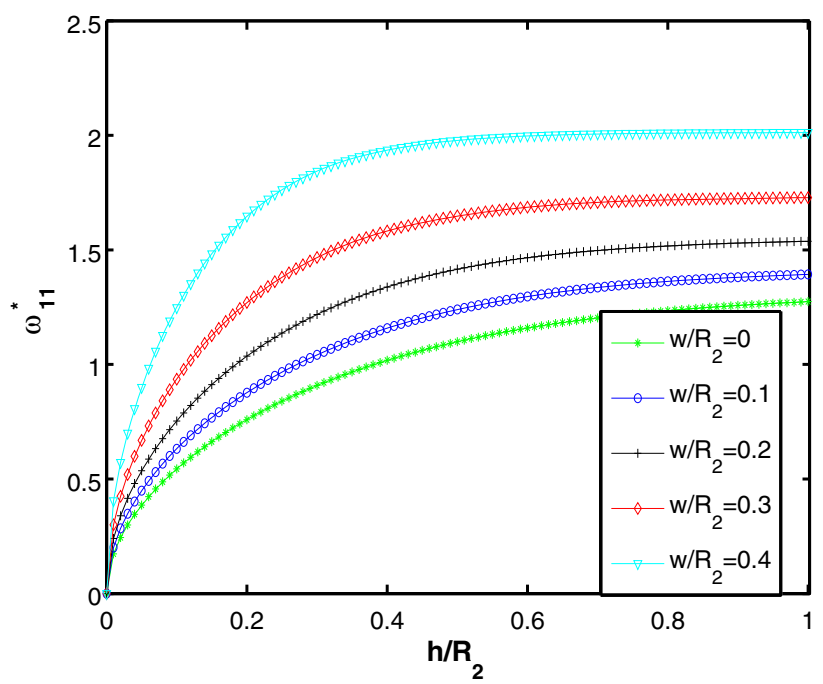

Figure 7. Sloshing frequency $\omega_{11}^{*}(m=1, n=1)$ for different annular baffles of width $\left(w=\left(\frac{2}{3}-\alpha\right) R_{2}\right)$ vs height ratio $h / R_{2}$ without surface tension $\sigma$.

\subsection{Case II: $\quad R_{2} / R_{1}=3$, relevant upper surface conditions and numerical results}

Here we consider the radius of the inner cylinder as one third of that of the outer cylinder, i.e., in this case $\alpha \in\left(0, \frac{2}{3}\right]$. Equations (12) and (13) are solved for $R_{2} / R_{1}=$ 3 and $\alpha \in\left(0, \frac{2}{3}\right]$.

Here also we determine the dimensionless fundamental sloshing frequency as function of liquid height ratio $h / R_{2}$ for a fixed Bond number and for various $\alpha$ values as shown in figure 6 . The curves shown in figure 6 are plotted for the

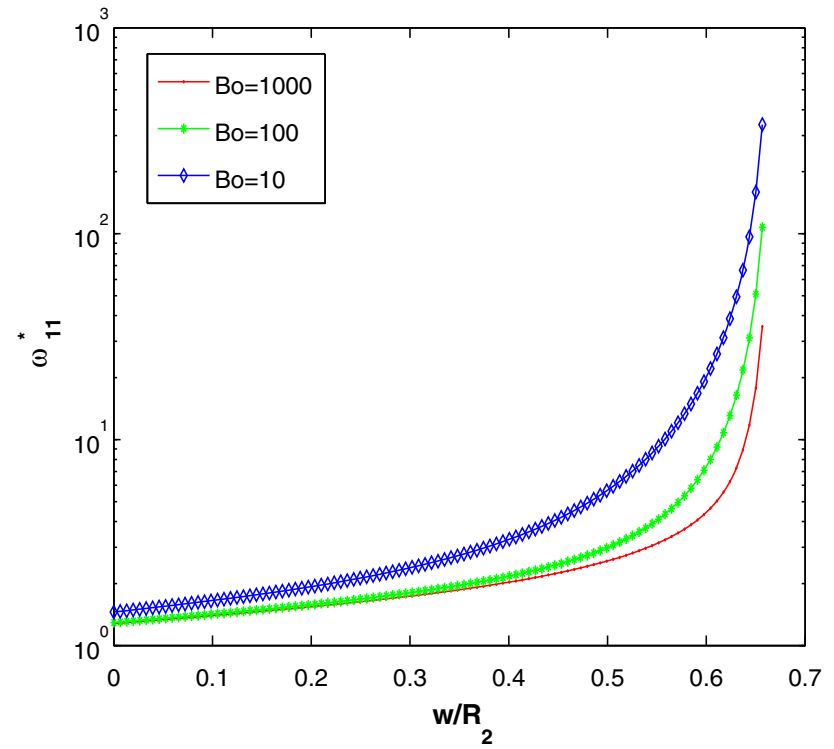

Figure 8. Effect of baffle width $w / R_{2}=\left(\frac{2}{3}-\alpha\right)$ on $\omega_{11}^{*}(m=$ $1, n=1)$ at height ratio $h / R_{2}=1$ for different Bond numbers.

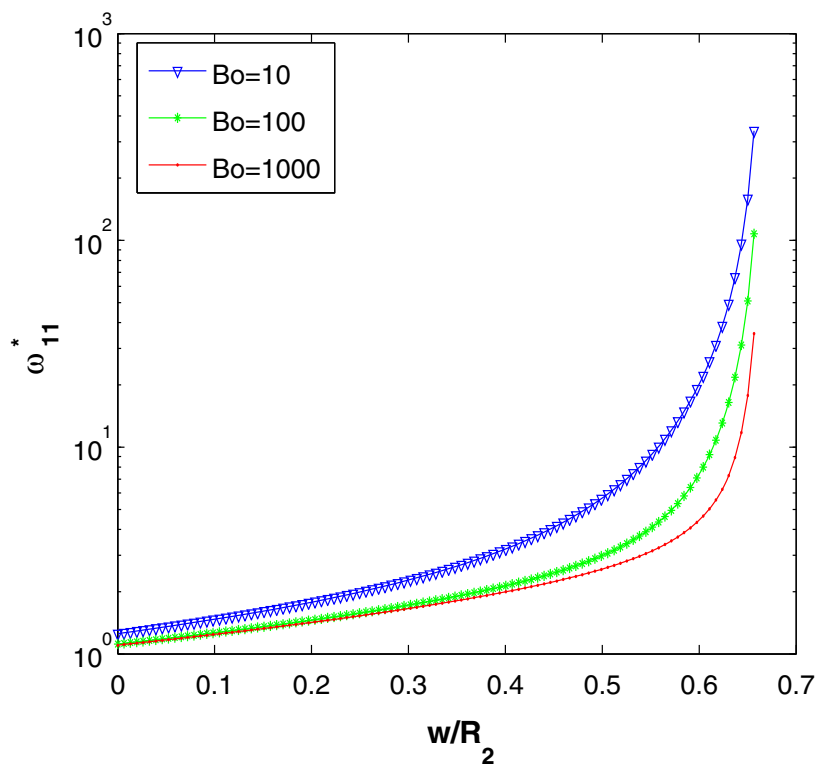

Figure 9. Effect of baffle width $w / R_{2}=\left(\frac{2}{3}-\alpha\right)$ on $\omega_{11}^{*}(m=$ $1, n=1)$ at height ratio $h / R_{2}=\frac{1}{2}$ for different Bond numbers.

lowest sloshing mode $\omega_{11}^{*}(m=1, n=1)$. Dimensionless fundamental sloshing frequency $\omega_{11}^{*}$ increases smoothly with increasing height ratio $h / R_{2}$ for various $\alpha$ values and fixed Bond number $B o=100$.

A baffle with width of about $w=0.4 R_{2}$ doubles the fundamental natural frequency. Frequency attains higher values corresponding to higher values of height ratio $h / R_{2}$. In comparison with figure 2 , where $R_{2} / R_{1}=2$, here the values of natural frequency are smaller, which implies that the sloshing mass will be higher. In the earlier case the 


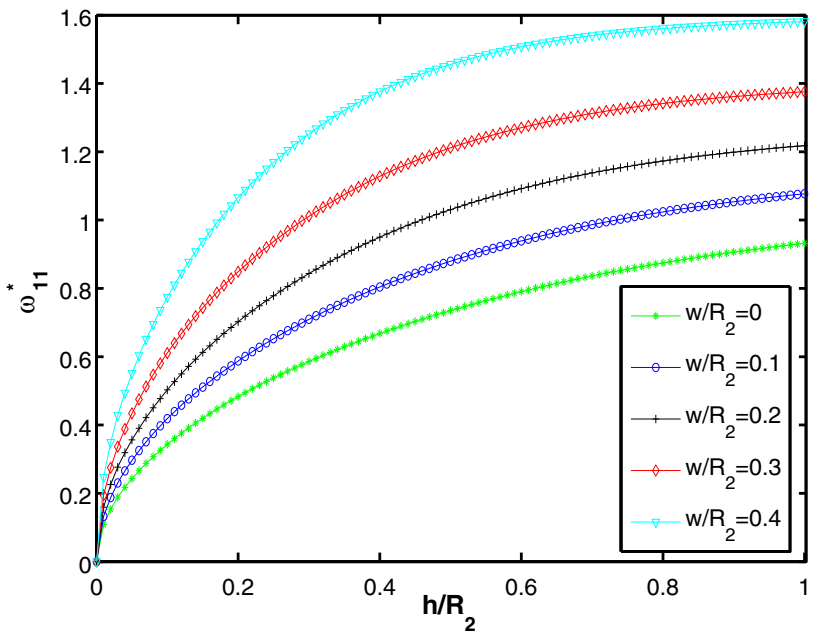

Figure 10. Sloshing frequency $\omega_{11}^{*}(m=1, n=1)$ for different annular baffles of width $\left(w=\left(\frac{3}{4}-\alpha\right) R_{2}\right)$ vs height ratio $h / R_{2}$ for $B o=100$

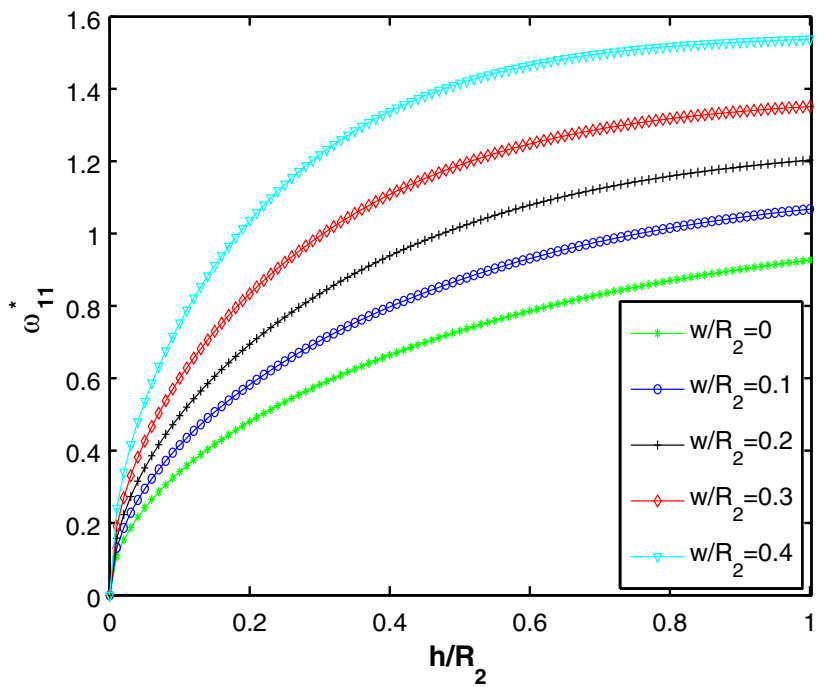

Figure 11. Sloshing frequency $\omega_{11}^{*}(m=1, n=1)$ for different annular baffles of width $\left(w=\left(\frac{3}{4}-\alpha\right) R_{2}\right)$ vs height ratio $h / R_{2}$ without surface tension $\sigma$.

frequencies doubled even for an increase of baffle width 0.1 whereas in this case this doubling occurs when an increase of $w / R_{2}=0.4$ takes place.

Figure 7 shows the curves drawn for $\omega_{11}^{*}$ as a function of liquid height ratio $h / R_{2}$ for various $\alpha$ values in which surface tension $\sigma$ is assumed to be absent. It is observed that for various $\alpha$ values, the effect of baffle width remains the same, resulting in reduced sloshing frequency. In the absence of surface tension, sloshing is observed to be more. It is observed that frequency reduces in this case, i.e., without surface tension the natural frequency reduces in comparison with the case when $B o=100$.

In figures 8 and 9 , we show the dimensionless fundamental sloshing frequency as a function of the magnitude of

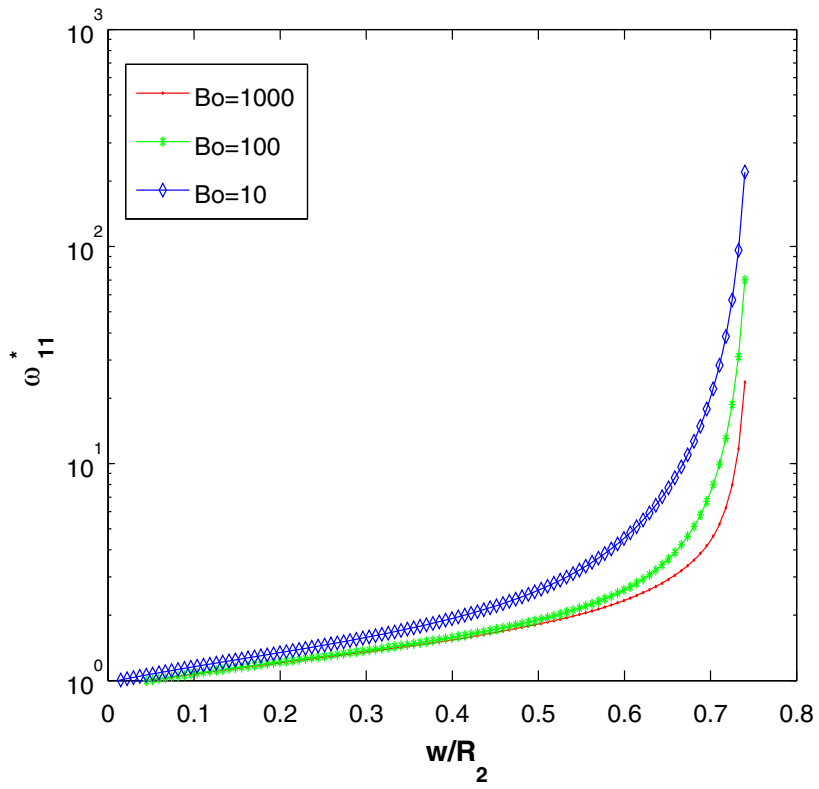

Figure 12. Effect of baffle width $w / R_{2}=\left(\frac{2}{3}-\alpha\right)$ on $\omega_{11}^{*}(m=$ $1, n=1)$ at height ratio $h / R_{2}=1$ for different Bond numbers.

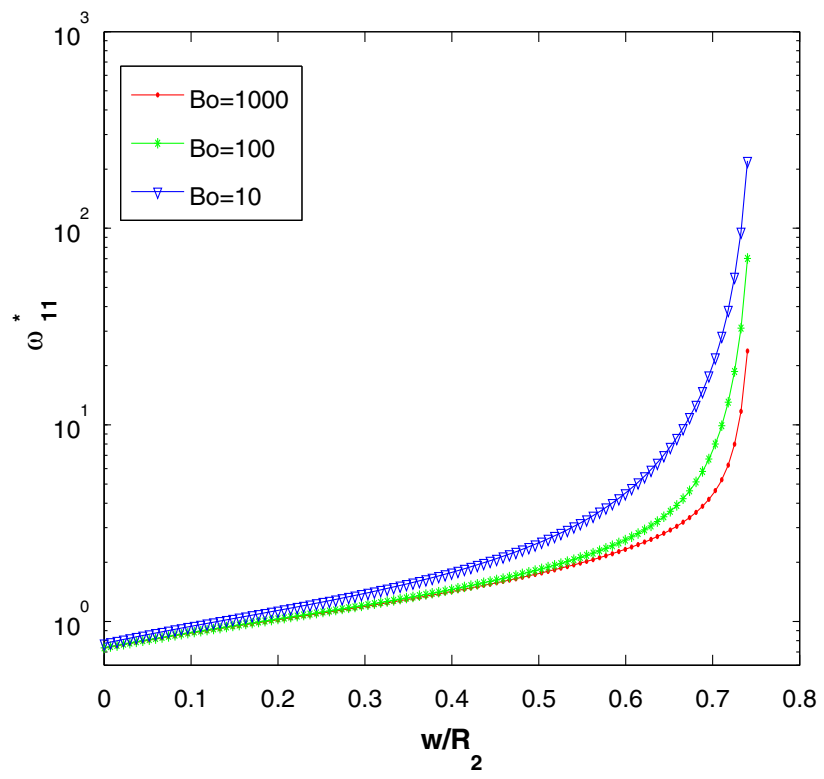

Figure 13. Effect of baffle width $w / R_{2}=\left(\frac{2}{3}-\alpha\right)$ on $\omega_{11}^{*}(m=$ $1, n=1)$ at height ratio $h / R_{2}=\frac{1}{2}$ for different Bond numbers.

the annular baffle width $w / R_{2}=2 / 3-\alpha$ for fixed liquid height ratio $h / R_{2}=1,1 / 2$, respectively, for different Bond numbers $B o=10,100,1000$. Here it is observed that frequency increases when the value of Bond number decreases, i.e., when surface tension increases. Figures 6-9 establish that frequency increases with increasing baffle width, increasing height ratio and with decreasing Bond number. 


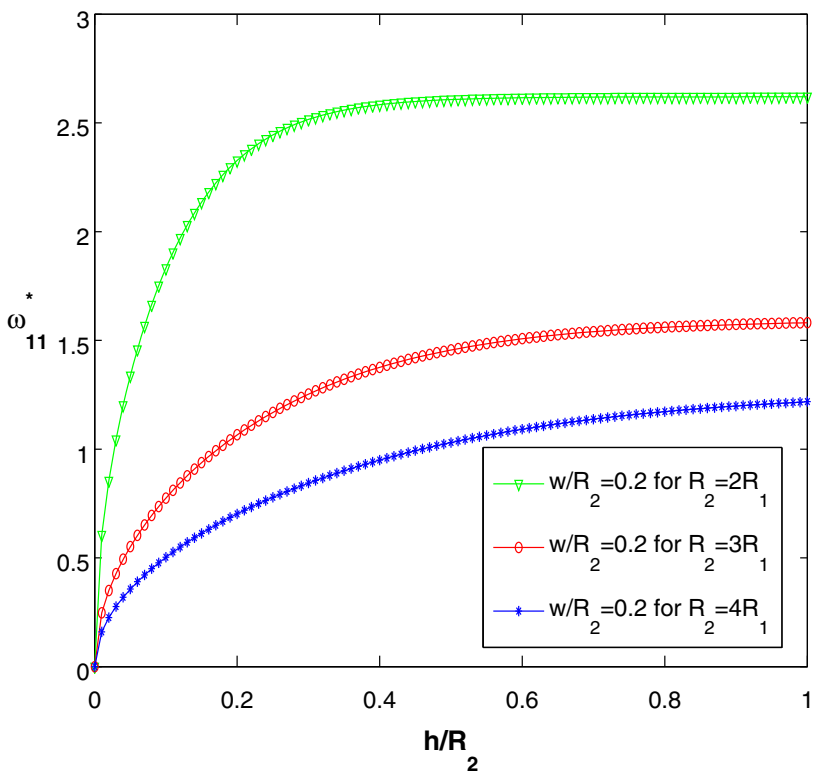

Figure 14. Sloshing frequency $\omega_{11}^{*}(m=1, n=1)$ for different cases with an annular baffle of width $\left(w=0.2 R_{2}\right)$ vs height ratio $h / R_{2}$ for $B o=100$.

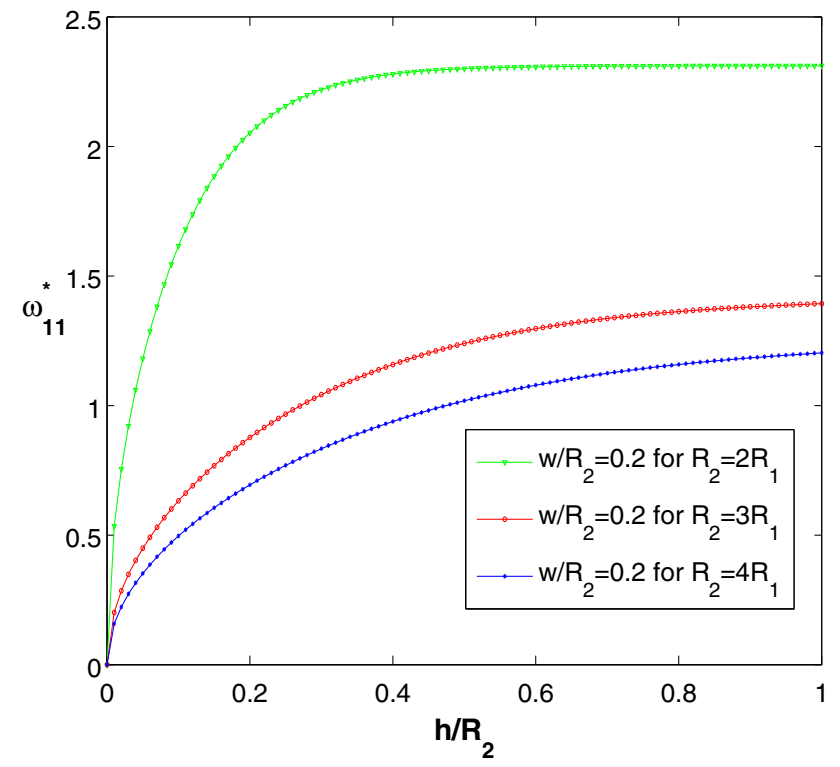

Figure 15. Sloshing frequency $\omega_{11}^{*}(m=1, n=1)$ for different cases with an annular baffle of width $\left(w=0.2 R_{2}\right)$ vs height ratio $h / R_{2}$ without surface tension $\sigma$.

\subsection{Case III: $R_{2} / R_{1}=4$, relevant upper surface conditions and numerical results}

Here we consider the radius of the inner cylinder as one fourth of that of the outer cylinder, i.e., in this case $\alpha \in\left(0, \frac{3}{4}\right]$. Equations (12) and (13) are solved for $R_{2} / R_{1}=4$ and $\alpha \in\left(0, \frac{3}{4}\right]$.

Here we again determine the dimensionless fundamental sloshing frequency as a function of liquid height ratio $h / R_{2}$

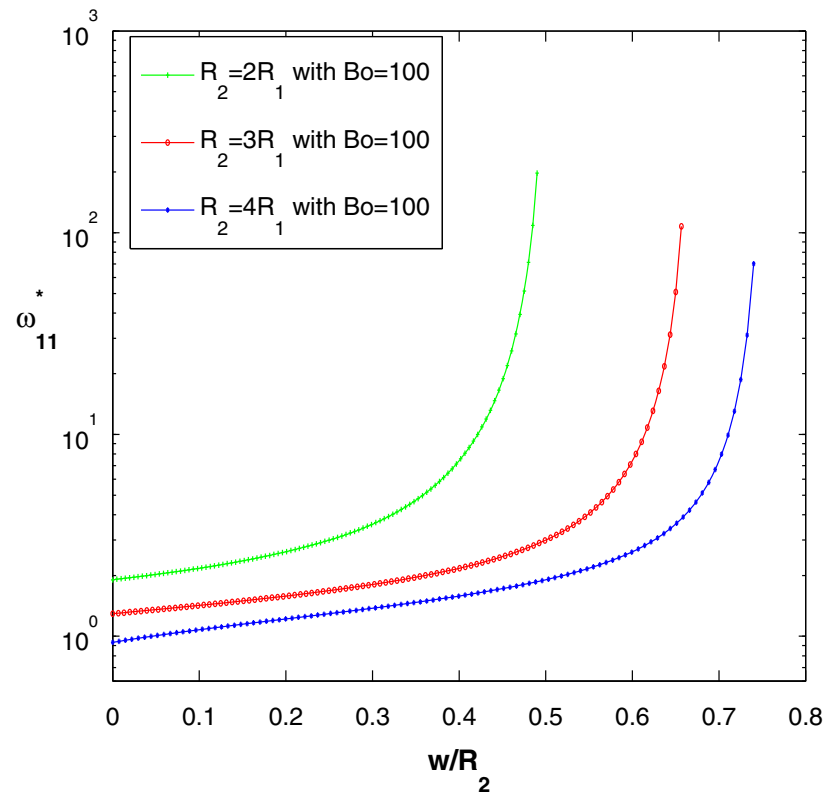

Figure 16. Effect of baffle width $w / R_{2}=(1-\alpha)-\frac{R_{1}}{R_{2}}$ on $\omega_{11}^{*}(m=1, n=1)$ at height ratio $h / R_{2}=1$ for Bond number $B o=100$.

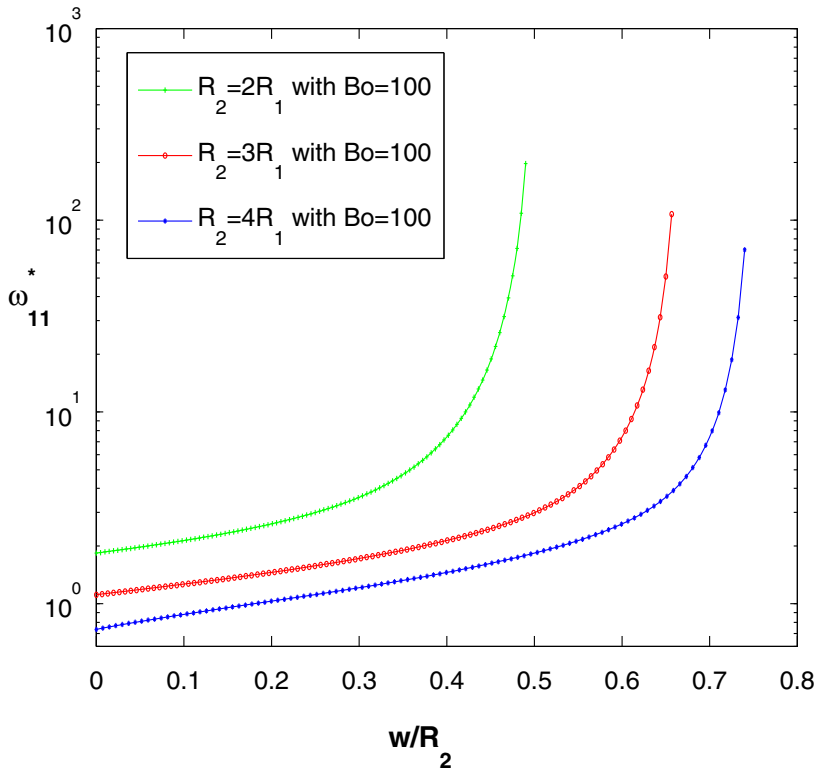

Figure 17. Effect of baffle width $w / R_{2}=(1-\alpha)-\frac{R_{1}}{R_{2}}$ on $\omega_{11}^{*}(m=1, n=1)$ at height ratio $h / R_{2}=\frac{1}{2}$ for Bond number $B o=100$.

for a fixed Bond number and for various $\alpha$ values, as shown in figure 10. The curves shown in figure 10 are plotted for the lowest sloshing mode $\omega_{11}^{*}(m=1, n=1)$, which exhibits the largest sloshing mass participating in the motion. Dimensionless fundamental sloshing frequency $\omega_{11}^{*}$ 


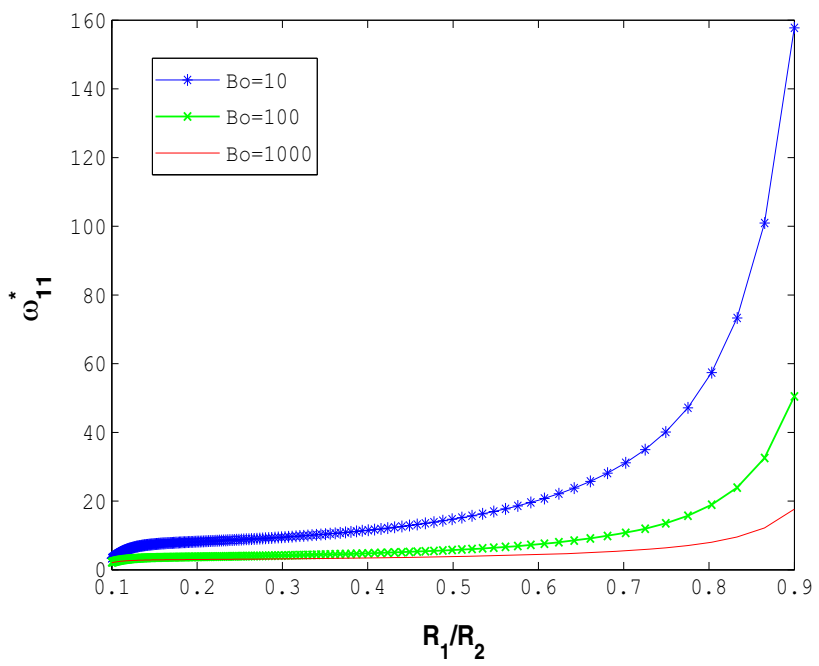

Figure 18. Effect of inner-outer radii ratio $R_{1} / R_{2}$ on $\omega_{11}^{*}(m=$ $1, n=1)$ at height ratio $h / R_{2}=1$ for different Bond numbers.

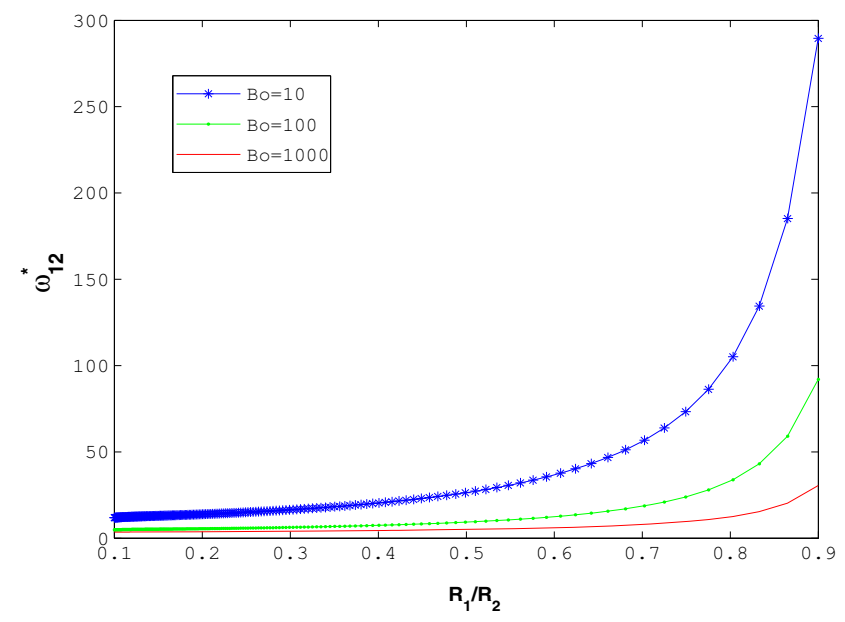

Figure 19. Effect of inner-outer radii ratio $R_{1} / R_{2}$ on $\omega_{12}^{*}(m=$ $1, n=2)$ at height ratio $h / R_{2}=1$ for different Bond numbers.

Table 1. Root comparison with first three roots calculated in Bauer [23] for $m=1$; Source Ibrahim [21].

\begin{tabular}{|c|c|c|c|c|c|}
\hline \multicolumn{2}{|c|}{$\frac{R_{1}}{R_{2}}=0.01$} & \multicolumn{2}{|c|}{$\frac{R_{1}}{R_{2}}=0.1(\alpha=0.9)$} & \multicolumn{2}{|c|}{$\frac{R_{1}}{R_{2}}=0.2$} \\
\hline Our & Bauer & Our & Bauer & Our & Bauer \\
\hline 1.8408 & 1.8408 & 1.8035 & 1.8034 & 1.7051 & 1.7051 \\
\hline 5.3291 & 5.3291 & 5.1371 & 5.1371 & 4.9608 & 4.9608 \\
\hline 8.5305 & 8.5305 & 8.1992 & 8.1991 & 8.4331 & 8.4330 \\
\hline
\end{tabular}

increases rapidly with increasing height ratio $h / R_{2}$ for various $\alpha$ values and fixed Bond number $B o=100$.

A baffle with width of about $w=0.4 R_{2}$ doubles the fundamental natural frequency, i.e., for the case when $w / R_{2}=0$. Frequency changes to higher values corresponding to the higher values of height ratio $h / R_{2}$.

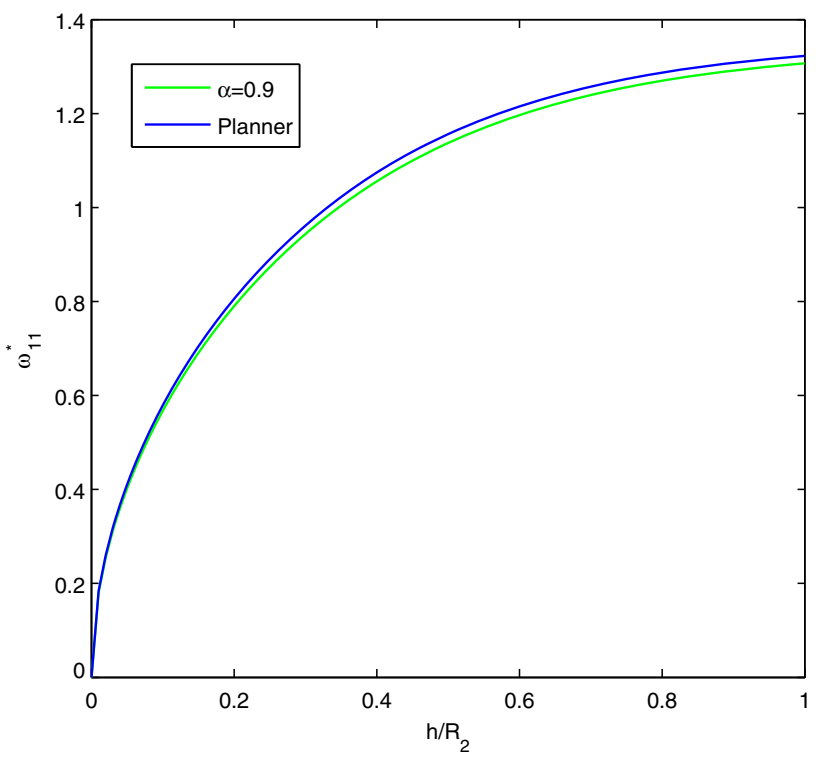

Figure 20. Sloshing frequency $\omega_{11}^{*}(m=1, n=1)$ without surface tension.

Frequency also increases continuously with increasing values of baffle width.

Figure 11 shows the curves drawn for $\omega_{11}^{*}$ as a function of liquid height ratio $h / R_{2}$ for various $\alpha$ values in which surface tension $\sigma$ is assumed to be absent. It is observed that for various $\alpha$ values, the effect of baffle width remains the same, resulting in reduced sloshing frequency. In the absence of surface tension, sloshing is observed to be more. For the baffle width $w=0.4 R_{2}, \omega_{11}^{*}$ is twice as compared with the value when there is no baffle on the free surface. It is observed that frequency reduces without surface tension.

In figures 12 and 13, we show the dimensionless fundamental sloshing frequency as a function of the magnitude of the annular baffle width $w / R_{2}=3 / 4-\alpha$ for fixed liquid height ratio $h / R_{2}=1,1 / 2$, respectively, for different Bond numbers $B o=10,100,1000$. Here it is observed that frequency increases when the value of Bond number decreases, i.e., when surface tension increases. Figures 10-13 establish that frequency increases with increasing baffle width, increasing height ratio and with decreasing Bond number.

\subsection{Comparison of all cases}

We want to sum up the effect of the sloshing frequency due to the changing fluid domain for different $R_{2} / R_{1}$ ratios.

In figure 14, curves are drawn for the fixed baffle width $w / R_{2}=0.2$ for various cases of $R_{2} / R_{1}$. For the case $R_{2}=2 R_{1}$, frequency is more than twice as compared with the frequency for $R_{2}=4 R_{1} ; \omega_{11}^{*}$ is nearly twice for $R_{2}=$ $2 R_{1}$ as compared with $\omega_{11}^{*}$ for $R_{2}=3 R_{1}$. It is observed that for $R_{2}=2 R_{1}$, frequency becomes stable as liquid height 
ratio $h / R_{2}$ increases, which is not significant in the other two cases. After comparing all three cases, sloshing is observed to be more when free surface is wider for a fixed baffle width. That is, sloshing is more when $R_{2}$ is much greater than $R_{1}$. In this case, the best option is to increase the baffle width so as to reduce the free surface and also to have the container filled with more volume of liquid at the same time.

Figure 15 shows the curves drawn for $\omega_{11}^{*}$ as a function of liquid height ratio $h / R_{2}$ for fixed baffle width $w / R_{2}=$ 0.2 for various cases that are considered, in which surface tension $\sigma$ is assumed to be absent. It is observed that the effect of baffle width remains the same, resulting in reduced sloshing frequency. In the absence of surface tension, sloshing is observed to be more.

In figures 16 and 17, curves are drawn for all cases, for fixed height ratio $h / R_{2}=1,1 / 2$, respectively, corresponding to a fixed Bond number $B o=100$. For the case $R_{2}=2 R_{1}$, frequency is much higher as compared with the other cases. For the case $R_{2}=3 R_{1}, \omega_{11}^{*}$ is higher than that for the case $R_{2}=4 R_{1}$. Curves show that for fixed height ratio $h / R_{2}$, frequency increases as baffle width increases. For $R_{2}=2 R_{1}$, frequency increases at a very high rate as compared with the other cases. For wider free surface, sloshing is observed to be more. Figures 14-17 show that frequency increases with increasing baffle width as well as with increasing height ratio, i.e., frequency increases with decreasing free surface. These results suggest the use of a cylindrical container so that sloshing is less but at the same time more fluid can be filled.

In figures 18 and 19, we show the dimensionless fundamental sloshing frequency as a function of the magnitude of the inner-outer radii ratio $R_{1} / R_{2}$ for fixed liquid height ratio $h / R_{2}=1$, for different Bond numbers $B o=10,100,1000$ in case of no baffle at free surface. Here it is observed that when radii ratio increases, nondimensional frequency increases. It is shown that frequency can be increased by decreasing the free surface. It is also shown that second frequency in first mode is much higher compared with first frequency. Here also it is observed that frequency increases when the value of Bond number decreases, i.e., when surface tension increases.

We have compared our computations for first three roots for $m=1$ determined by Eq. (11) with roots determined by Bauer [23] (table 1). We have calculated for $\frac{R_{1}}{R_{2}}=0.1$. In figure 20, non-dimensional frequency $\omega_{11}$ is plotted for $\alpha=$ 0.9 and for a cylindrical case. A good agreement is found in both the cases, which validates our results.

It is to be noted that the free surface and its baffle conditions were usually satisfied at $N_{1}+N_{2}=10$ equidistantly distributed points. Further, if this whole investigation is carried out by placing the baffle on the outside wall of the inner cylinder, it is still possible to obtain similar results.

\section{Conclusion}

In this work, we consider a circular cylindrical container of specific size in which fluid is filled in the annular region of the container. We introduce an annular baffle mounted on the wall of the outer cylinder at the free surface. We investigate the sloshing frequencies for different baffle width, fluid height and radial values of the annular region. Results show the dependence of sloshing frequencies on baffle width as well as on liquid height ratio. It is shown that fundamental sloshing frequency increases when baffle width increases. It is clear that when an annular baffle is introduced at the container wall in the free liquid surface plane, it yields increased natural frequencies. Partial coverage of the free surface has a beneficial effect on the system. It is also shown that in the presence of surface tension, frequency increases. Three different cases are solved numerically for increasing ring baffle with fixed Bond number $B o=100$. We also investigate the sloshing frequencies against baffle width for different Bond numbers. Fundamental sloshing frequency increases gradually as baffle width and liquid height ratios increase. It is shown that the liquid sloshing frequency inside the container decreases with increasing baffle width for a constant height ratio $h / R_{2}=1$. As the natural frequency increases, the sloshing mass decreases, thereby bringing stability to the overall structure, including the vehicle in which the liquid container is being transported. In other words, the annular baffle plays a very important role on the sloshing frequency of liquid. It is found that even for larger radius values for the fluid domain, sloshing can be controlled by inserting wider annular baffles accordingly. The method presented here is expected to be easily applicable to any structural system in the free surface plane.

\section{Acknowledgements}

The first author wishes to thank Indian Institute of Technology Guwahati for providing her graduate fellowship to pursue $\mathrm{PhD}$. Both authors are grateful to the esteemed Reviewer and the Editor-in-Chief for allowing revision of the manuscript.

\section{References}

[1] Ockendon H, Ockendon J R and Johnson A D 1986 Resonant sloshing in shallow water. J. Fluid Mech. 167: 465-479

[2] Evans D V 1990 The wide spacing approximation applied to multiple scattering and sloshing problems. J. Fluid Mech. 210: 647-658

[3] Rocca M L, Sciortino G and Boniforti M A 2000 A fully nonlinear model for sloshing in a rotating container. Fluid Dyn. Res. 27: 23-52 
[4] Faltinsen O M, Rognebakke O F, Lukovsky I A and Timokha A N 2000 Multidimensional modal analysis of nonlinear sloshing in a rectangular tank with finite water depth. J. Fluid Mech. 407: 201-234

[5] Faltinsen O M and Timokha A N 2001 An adaptive multimodal approach to nonlinear sloshing in a rectangular tank. J. Fluid Mech. 432: 167-200

[6] Faltinsen O M and Timokha A N 2002 Asymptotic modal approximation of nonlinear resonant sloshing in a rectangular tank with small fluid depth. J. Fluid Mech. 470: 319-357

[7] Faltinsen O M, Rognebakke O F and Timokha A N. 2003 Resonant three-dimensional nonlinear sloshing in a squarebase basin. J. Fluid Mech. 487: 1-42

[8] Faltinsen O M, Rognebakke O F and Timokha A N 2005 Resonant three-dimensional nonlinear sloshing in a square-base basin. Part 2. Effect of higher modes. J. Fluid Mech. 523: 199-218

[9] Thiagarajan K P, Rakshit D and Repalle N 2011 The air water sloshing problem: fundamental analysis and parametric studies on excitation and fill levels. Ocean Eng. 38: 498-508

[10] Mackey D and Cox E A 2003 Dynamics of two-layer fluid sloshing problem. IMA J. Appl. Math. 68: 665-686

[11] McIver P and McIver M 1993 Sloshing frequencies of longitudinal modes for a liquid contained in a trough. J. Fluid Mech. 252: 525-541

[12] Bauer H F and Eidel W 1999 Frictionless liquid sloshing in circular cylindrical container configurations. Aerosp. Sci. Technol. 3: 301-311

[13] Papaspyrou S, Karamanos S A and Valougeorgis D 2004 Response of half full horizontal cylinders under transverse excitations. J. Fluid Struct. 19: 985-1003

[14] Gavrilyuk I, Lukovsky I, Trotsenko Yu and Timokha A 2007 Sloshing in a vertical circular cylindrical tank with an annular baffle. Part 2. Nonlinear resonant waves. J. Eng. Math. 57: 57-78

[15] He Y, Ma X, Wang P and Benli W 2007 Low-gravity liquid nonlinear sloshing analysis in a tank under pitching excitation. J. Sound Vib. 299: 164-177

[16] Sinai Y L 1985 Fundamental sloshing frequencies of stratified two fluid system in closed prismatic tanks. Int. J. Heat Fluid Fl. 6: 142-144

[17] Sciortino G, Adduce C and Rocca M L 2009 Sloshing of a layered fluid with a free surface as a Hamiltonian system. Phys. Fluids 21: 052102

[18] Albright N and Concus P 1977 Small amplitude periodic sloshing modes of a liquid in a vertical right circular cylinder with a concave spheroidal bottom. Report, U.S. Department of Energy, 84

[19] Bian X, Perlin M, Schultz W W and Agarwal M 2003 Axisymmetric slosh frequencies of a liquid mass in a circular cylinder. Phys. Fluids 15: 3659-3664

[20] Gavrilyuk I P, Lukovsky I A and Timokha A N 2005 Linear and nonlinear sloshing in a circular conical tank. Fluid Dyn. Res. 37: 399-429

[21] Ibrahim R A 2005 Liquid sloshing dynamics: theory and applications. Cambridge University Press

[22] Sorolla E, Mosig J R and Mattes M 2013 Algorithm to calculate a large number of roots of the cross-product of Bessel functions. IEEE Trans. Antennas Propag. 61: 2180-2187

[23] Bauer H F 1963 The Effect of Propellant sloshing on the stability of an accelerometer controlled rigid space vehicle, National Aeronautics and Space Administration Report, George C. Marshall Space Flight Center, Huntsville, Alabama, 94 pages 